\section{Re: Aggressive Surgical Approach for Patients with T4 Gastric Carcinoma: Promise or Myth?}

\section{TO THE EDITORS:}

We read the article by Cheng et al. ${ }^{1}$ in January issue of Annals of Surgical Oncology with interest. They analyzed a cohort of 179 patients with advanced gastric cancer who underwent gastrectomy with curative intent, and they asserted that aggressive surgical management of $\mathrm{T} 4$ gastric cancer should be limited to patients without adverse prognostic factors, such as advanced nodal involvement and pancreatic invasion. However, we worried about some confusion made by the mixed-up usage of the pathologic terminology in terms of the classification of $\mathrm{T}$ stage, especially regarding the "pT4" group. According to the sixth edition of American Joint Committee on Cancer Staging System (AJCC), T4 designated as "the tumor has penetrated the peritoneal lining or serosa of the stomach and invaded the adjacent organ." In this study, Cheng et al. described that among 91 patients with pT4 treated with multiple organ resection (MOR) only 18 patients (19\%) had pathologically proven cancer cell infiltration in resected organs. Based on this result, the other 73 patients who were proven not to have adjacent organ infiltration with cancer cell should be classified as pT3 not pT4 based on sixth AJCC. Here, the authors might use the pT4 as in the seventh AJCC classification. We agreed that in cases of clinically T4 (cT4) gastric cancer based on preoperative evaluation, it is not always easy to discern whether it invades adjacent organ.
Secondly, Cheng et al. proposed that radical gastrectomy with combined MOR should be applied to patients without advanced nodal involvement and pancreatic invasion. In our opinion, the advanced nodal involvement could be inevitably associated with advanced disease status of the main mass lesion of the stomach. So, it is not appropriate to say that advanced nodal involvement itself should be regarded as exclusion criteria for aggressive surgical approach. Also, pancreatic invasion may impose the morbidity or mortality, such as pancreatic fistula, bleeding, or leakage. But it is rather associated with the preoperative performances of the patient not with the advanced disease status.

We proposed that Cheng et al. must clarify their definition of pT4 because of the mixed-up application of the sixth or seventh AJCC classification. In this report, we could probably draw a conclusion as such; in some cT4 patients, they could get survival benefit by the aggressive surgical approach if they are proven to have pT3 disease.

Jung Ho Shim, MD, Seong Il O, MD, and Kyo Young Song, MD, PhD

Department of Surgery, Seoul St. Mary's Hospital, College of Medicine, The Catholic University of Korea, Seoul, Korea

e-mail: skygs@catholic.ac.kr

Published Online: 2 July 2011

(C) Society of Surgical Oncology 2011

\section{REFERENCE}

1. Cheng CT, Tsai CY, Hsu JT, Vinayak R, Liu KH, Yeh CN, Yeh TS, Hwang TL, Jan YY. Aggressive surgical approach for patients with T4 gastric carcinoma: promise or myth? Ann Surg Oncol. 2011;18:1606-14. 